\title{
Original $\mid$ A FORWARD PLANNING INTENSITY-MODULATED RADIATION THERAPY Article WITH SIMULTANEOUS INTEGRATED BOOST IN THE TREATMENT OF HEAD AND NECK CANCER
}

Hussein Omar and Ahmed Habash

King Abdul Aziz Hospital and Oncology Center, Jeddah, Saudi Arabia

\begin{abstract}
Introduction: The aim of this study is to describe in detail forward planning intensity-modulated radiation therapy (FPIMRT) technique used in our center for the treatment of head-and-neck cancer and to confirm that in centers without the capabilities of inverse planning intensity-modulated radiation therapy (IP-IMRT) or in situations where patients are not ideal candidates for that technique, FP- IMRT can be useful and can achieve a good dose distribution with sparing of parotid glands.
\end{abstract}

Patients and Methods: Between June 2007 and April 2008, 19 patients with primary head-and-neck cancers were treated at King Abdul Aziz Hospital and Oncology Center Jeddah, using FP-IMRT techniques. In our study we follow the RTOG H-0022 protocol, thirty daily fractions over six weeks are used to simultaneously deliver 66Gy (2.2Gy/fraction) to the gross tumor (primary and enlarged nodes) with margins based on radiological and clinical justification (clinical target volume 1), 60Gy (2Gy/fraction) to the soft tissue and nodal regions adjacent to the previous volume (clinical target volume 2) and 54Gy (1.8Gy/fraction) to elective nodal regions as well as margin to account for patient motion and setup errors (clinical target volume3). These are biologically equivalent to 70, 60 and 50Gy, respectively, if given in 2Gy per fraction. The maximum dose to the brainstem and spinal cord are to be maintained below 54 and 50Gy, respectively. The mean dose to the parotid is to be maintained below $26 \mathrm{~Gy}$, or at least $50 \%$ of one of the parotid is to be maintained below 30Gy. All patients treated with a 7 gantry angles, including an anterior, 2 lateral, 2 anterior oblique and 2 posterior oblique angles with a total of 22 beam shapes formed by multileaf collimators (MLC). The segments used in a given angle are tailored to maximize the coverage of the target while minimizing the normal tissue exposure. All patients were treated with 6MV photon produced by Siemens (Oncor Impression) accelerator with autosequencing of all treatment segments. Results: The aim of having at least $95 \%$ and $98 \%$ of the volume of CTV1 to receive at least $95 \%$ and $90 \%$ of the prescribed dose, respectively, was fulfilled in all cases. The criterion of having at least $95 \%$ and $98 \%$ of the volume of CTV2 to receive at least $95 \%$ and $90 \%$ of the prescribed dose was not met in five and six patients, respectively, with an average of $92.8 \%$ of the volume received $95 \%$ of the dose and $97.3 \%$ of the volume received $90 \%$ of the dose. On the other hand the percentage of the volume of CTV3 who received $95 \%$ of dose had a wide range from $81 \%$ to $99 \%$ with an average of $88.3 \%$ and the percentage that received $90 \%$ of the dose ranged from $85 \%$ to $100 \%$ with an average of $94.5 \%$. The maximum dose to the spinal cord was ranged from 50 to 39Gy (average $45.1 \mathrm{~Gy}$ ), however the dose to $10 \%$ of the spinal cord volume present in the field ranged from 47 to 30Gy (average 39.3Gy). On the other hand the dose to the brain stem was below 54Gy in all cases. We succeed to keep the mean dose of both parotid glands below 26Gy in four patients, and the other fifteen patients have at least one parotid gland with a mean dose below 26Gy.

Conclusion: The forward-planning intensity-modulated radiation therapy (FP- IMRT) allows the delivery of a high dose to the target coverage while minimizing the normal tissue toxicity. In a center without the capabilities of IP-IMRT or in situations where patients are not ideal candidates for that technique, FP- IMRT can be useful and can achieve a good dose distribution.

Key Words: Head and neck cancer IMRT.

Corresponding Author: Hussein Omar, Tel. : 0100004551, E-mail: hussainomar@hotmail.com

\section{INTRODUCTION}

During the second half of last century, key technological innovations have tremendously modified the daily practice of radiotherapy, leading to substantial improvements in treatment delivery and outcome ${ }^{1}$.

In the late 1990s, the availability of advanced treatment planning systems together with multileaf collimators have progressively contributed to a more targeted and conformed dose delivery, that is, a better dose distribution within the target volumes while sparing surrounding normal tissues. In addition, linacs are nowadays equipped with electronic portal imaging devices for verification of patient positioning, thus enabling a better conformity between the planned dose and the dose that is actually delivered ${ }^{1}$. 
The introduction of intensity-modulated radiation therapy (IMRT), over the last few years has brought another refinement in the ballistics of dose delivery, enabling further improvement in dose delivery and treatment outcome. IMRT is not only a technique for delivering optimized nonuniform beam intensities to a target volume, but it also provides a new approach to the whole treatment procedure from patient immobilization to beam delivery ${ }^{2}$.

Two approaches of computerized treatment planning for step and shoot IMRT are generally applied: The first method is an extension of conventional treatment planning and in this paper referred to as forward planning intensity-modulated radiation therapy (FP-IMRT). Its definition of the segment shapes is performed manually. However, more than one segment is used from each beam direction. Afterwards, the weights of the segments are optimized manually or using a computer optimization algorithm to achieve the desired dose distribution ${ }^{3}$.

The second strategy, which we denote as inverse planning intensity-modulated radiation therapy (IPIMRT), usually starts with the optimization of fluence profiles from each beam direction by minimization of an objective function. Afterwards, sequencing transforms each optimised profile into a series of segments, which can be delivered with a multileaf collimator (MLC) ${ }^{4}$.

Each year, there are increasing numbers of head-andneck cancer patients around the world being treated with IMRT $^{5}$. IMRT for these tumor sites is considered to be a promising method to improve local control and/or reduce acute or late toxicity. In particular, xerostomia by sparing the parotid glands without compromising the coverage of the planning target volume (PTV) .

Although IP-IMRT is the most ideal treatment technique for head-and-neck cancer patients especially for some complex clinical situations, which require the use of many beam directions and segments ${ }^{7-10}$, this technology is not readily available in every radiotherapy clinic throughout the world. Several factors contribute to this, including economic and technical constraints. First, IP-IMRT for head-and-neck cancer involves complex planning and treatment delivery, requiring a dedicated team effort of physicists and dosimetrists. Second, the longer daily treatment time associated with IP-IMRT may be a hindrance for a busy clinic trying to accommodate all patients requesting IP-IMRT. Lastly, some patients are simply not ideal candidates for IP-IMRT, because of their inability to remain immobilized for a prolonged time during the course of treatment ${ }^{11}$.

On the other hand the clinical implementation of IMRT using forward planning is relatively easy, because it is closely related to conventional planning. Issues like quality assurance, time involved in planning and delivery are a logical extension of the experience obtained with conformal radiotherapy, but the drawback with FP-IMRT is that plan optimization is done using manual iteration by the planning dosimetrist or physicist and is heavily dependent on the experience of the planner. However, with proper beam placement and beam weighting, the number of iterations can be significantly reduced ${ }^{12}$.

The Fractionation scheme is somewhat unique to IMRT treatments, in which each of the target regions receives different doses per fraction such a treatment strategy has been called simultaneous integrated boost $(\mathrm{SIB})^{13}$.The SIB-IMRT strategy not only can produce superior dose distributions but also is an easier, more efficient, and perhaps a less error prone method of planning IMRT because it involves the use of the same plane for the entire course of treatment. It also avoids the problem of field matching and junctioning encountered in many multifield boost treatment strategies ${ }^{14}$.

The aim of this study is to describe in detail FP-IMRT technique used in our center for the treatment of headand-neck cancer and to confirm that in centers without the capabilities of IP-IMRT or in situations where patients are not ideal candidates for that technique, FP- IMRT can be useful and can achieve a good dose distribution with sparing of parotid glands.

\section{PATIENTS AND METHODS}

Between June 2007 and April 2008, 19 patients with primary head-and-neck cancers were treated at King Abdul Aziz Hospital and Oncology Center Jeddah, using FP-IMRT techniques. All were treated with a 7 gantry angles.

Patients 18 years or older with histologically confirmed non metastatic squamous cell carcinoma of the nasopharynx, oropharynx, hypopharynx, larynx, tonsil or oral cavity and were previously untreated were eligible for this trial. The disease was staged according to the 2002 classification of the American Joint Committee on cancer.

All patients underwent staging work up including physical examination, complete blood work, chest x-ray, abdominal ultrasound, computed tomography or magnetic resonance imaging of head and neck. Additional tests include fiberoptic triple endoscopy, bone scan, renogram and dental evaluation.

\section{Simulation and immobilization:}

For each patient, an initial simulation was done to establish the position, isocenter and immobilization of the patient. The patient's head was hyperextended or extended if the patient could not tolerate hyperextension.

Patients were then immobilized using a head or head-and-shoulder thermoplastic mask supported on a Timo neck support (Uni-Frame system, MED-TEC). 
The cervical spinal cord and brainstem were aligned as straight as possible. CT scans in serial 3-mm axial slices were obtained for treatment planning. CT images were acquired at least $6 \mathrm{~cm}$ beyond the cranial and caudal limits of the largest treatment target.

\section{Target and sensitive structure delineation:}

Treatment planning was done using the CMS planning system. Clinical target volume1 (CTV1), clinical target volume 2 (CTV2), clinical target volume 3 (CTV3) and the normal critical tissues, all were outlined on each axial CT slice. The CTV1 was defined as the gross tumor (primary and enlarged nodes) with margins based on radiological and clinical justification ${ }^{15}$. The CTV2 which defined as soft tissue and nodal regions adjacent to CTV1 and CTV3 which defined as elective nodal regions as well as margin to account for patient motion and setup errors. The normal critical structures included and evaluated were the brainstem, spinal cord and parotid glands.

\section{Fractionation scheme:}

One in the issues involved in the use of simultaneous integrated boost (SIB) technique is that each of the target regions receives different doses per fraction, which may create disproportionate corresponding biologic effects. However, the fraction sizes may be estimated by using an iso effective relationship based on linear-quadratic model and the values of its parameters (e.g., alpha-beta ratios and tumor doubling time).

In our study we follow the RTOG H-0022 protocol $^{14}$, thirty daily fractions over six weeks are used to simultaneously deliver 66Gy (2.2Gy/fraction) to CTV1, 60Gy (2Gy/fraction) to CTV2 and 54Gy (1.8Gy/fraction) to CTV3. These are biologically equivalent to 70,60 , and 50Gy, respectively, if given in 2Gy per fraction. The maximum dose to the brainstem and spinal cord are to be maintained below 54 and 50Gy, respectively. The mean dose to the parotid is to be maintained below 26Gy, or at least $50 \%$ of one of the parotid is to be maintained below $30 \mathrm{~Gy}$.

\section{Beam configuration:}

Figure (1) shows typical beam angle arrangements for seven beams, including an anterior, 2 laterals, 2 anterior oblique and 2 posterior oblique beams with a total of 22 beam shapes formed by multileaf collimators (MLC). Five of the 7 beam directions contained 20 segments (four segments for each) with equal weighting however the 2 lateral beams are not segmented. A single isocenter was used for all beams. The segments used in a given angle are tailored to maximize the coverage of the target while minimizing the normal tissue exposure. One can design, depending on the case, up to 4 segments at a given angle. For example, 4 segments are used at the left anterior oblique beam, at a gantry angle of $72^{\circ}$ (segments 5, 6, 7 and 8). The first segment was constructed to treat CTV1, CTV2 and CTV3 with a margin of $5 \mathrm{~mm}$ to account for penumbra, without shielding the spinal cord. The second segment was constructed to treat CTV1, CTV2 and CTV3 at one side of the spinal cord and the spinal cord was outside the field. The third segment was constructed to treat CTV1, CTV2 and CTV3 at the other side of the spinal cord and the spinal cord was outside the field also. The fourth segment was constructed to treat only the CTV1 with a 5-mm margin to account for penumbra without shielding the spinal cord. Similar principles were applied to the other beam directions except for the two lateral field, these two fields are not segmented and they are applied to compensate for the big separation of the head at the site of the primary tumor and it is tailored according to the dose distribution and both the spinal cord and the brainstem are shielded in these beams.

Once the typical beam arrangements were set, numerous adjustments and fine tuning were done to increase dose homogeneity and to decrease the dose delivered to the normal tissues. The shape of each MLC was carefully designed along with the associated weights. Manual iterations were done to optimize the plan.

Figure (2) show the different segment shapes superimposed on their associated digitally reconstructed radiographs for a case of nasopharyngeal carcinoma.

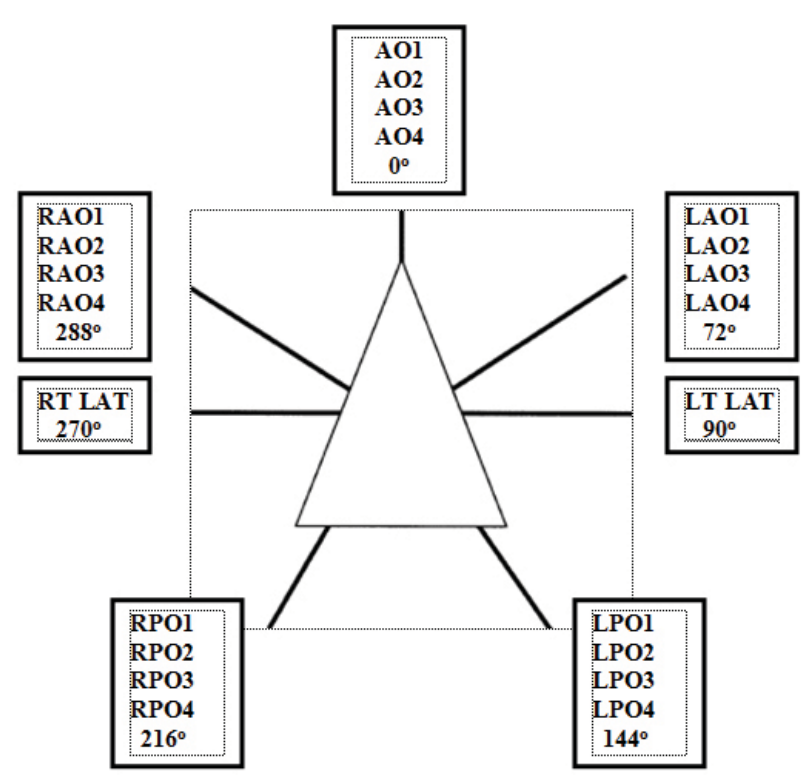

Fig. 1: Shows typical beam angle arrangements used in FP-IMRT. 


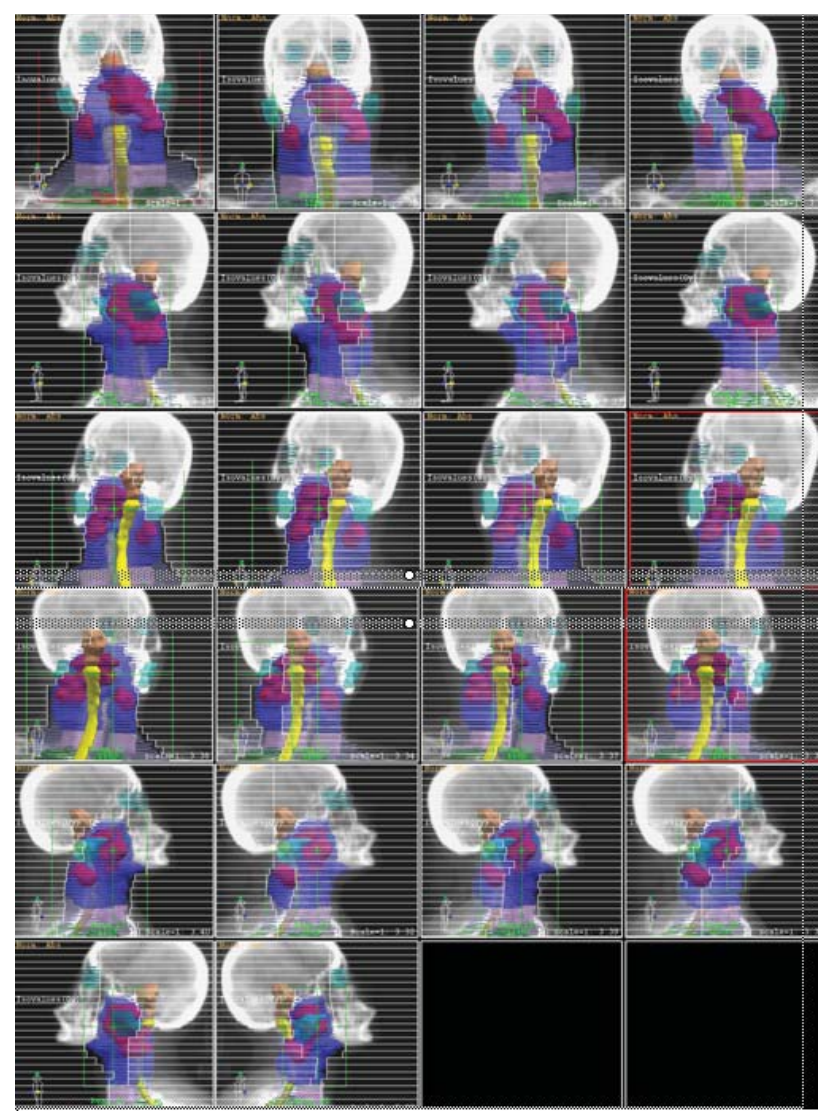

Fig. 2: Beam's-eye views of 22 digitally reconstructed radiographs for a case of nasopharyngeal carcinoma patient. Notice the different MLC shapes in each segment of a particular angle used in this plan.

\section{Plane approval:}

The aims of the treatment were to achieve a $90 \%$ of the prescribed dose to at least $98 \%$ of the volumes of CTV1, CTV2 and CTV3 and a dose of 95\% in at least 95\% of CTV1, CTV2 and CTV3. The maximum dose must be restricted to $107 \%$ of the prescribed dose. The maximum dose of the spinal cord must be limited to 50 Gy. Furthermore, the mean dose of either parotid gland should not exceed 26Gy or at least $50 \%$ of one of the parotid is to be maintained below 30Gy.

\section{Treatment delivery and quality assurance:}

At the start of radiotherapy, 22 films were taken to verify the treatment fields. Once all portal images matched exactly with the digitally reconstructed radiographs, weekly orthogonal portal films were taken to verify the treatment isocenter.

All patients were treated with 6MV photon produced by Siemens (Oncor Impression) accelerator with autosequencing of all treatment segments.

\section{Patient's assessment:}

All patients were assessed for response one month after completion of treatment. This assessment includes physical examination, CT scan or MRI of the primary and neck nodes. Endoscopy and biopsy was performed from any suspicious lesions.

\section{RESULTS}

Between June 2007 and April 2008 a total of 19 patients were recruited onto study. Patient characteristics, primary site distribution and primary and nodal staging are shown in (Table 1).

Table 1 : patient's characteristics.

\begin{tabular}{|c|c|c|}
\hline Characteristics & No of Patients & $\%$ \\
\hline \multicolumn{3}{|l|}{ Sex } \\
\hline Male & 13 & 68 \\
\hline Female & 6 & 32 \\
\hline \multicolumn{3}{|l|}{ Age, years } \\
\hline Median & 49 years & \\
\hline Range & 42-63 years & \\
\hline \multicolumn{3}{|l|}{$\begin{array}{l}\text { Primary site of the } \\
\text { tumor }\end{array}$} \\
\hline Nasopharynx & 9 & 48 \\
\hline Hypopharynx & 2 & 10.5 \\
\hline Larynx & 2 & 10.5 \\
\hline Oropharynx & 1 & 5 \\
\hline Tonsillar fossa & 1 & 5 \\
\hline Floor of mouth & 2 & 10.5 \\
\hline Base of tongue & 2 & 10.5 \\
\hline \multicolumn{3}{|l|}{ T-Stage } \\
\hline $\mathrm{T} 1$ & 1 & 5 \\
\hline $\mathrm{T} 2$ & 3 & 16 \\
\hline T3 & 12 & 63 \\
\hline $\mathrm{T} 4$ & 3 & 16 \\
\hline \multicolumn{3}{|l|}{ N-Stage } \\
\hline NO & 2 & 11 \\
\hline N1 & 7 & 37 \\
\hline N2 & 9 & 47 \\
\hline N3 & 1 & 5 \\
\hline \multicolumn{3}{|l|}{ Pathology } \\
\hline Differentiated & 9 & 47 \\
\hline Undifferentiated & 10 & 53 \\
\hline
\end{tabular}

\section{Treatment Compliance:}

Using an autosequencing delivery system, all Fields were treated automatically and successfully. The average treatment delivery time was $8 \mathrm{~min}$.

All 19 patients completed the intended treatment using FP-IMRT technique with simultaneous integrated boost however 2 patients had a gap of more than one week due to the development of Grade III and IV oropharyngeal mucositis (Both diagnosed as cancer tongue).

\section{Plan evaluation of target volume coverage and normal tissue sparing:}

Table (2a) and (2b) present the percentage of volume of CTV1, CTV2 and CTV3 received 95\% and 90\% of the prescribed dose, together with the maximum dose and the dose to ten percent of the spinal cord and the mean dose to the right and left parotid gland. 
The aim of having at least $95 \%$ and $98 \%$ of the volume of CTV1 to receive at least $95 \%$ and $90 \%$ of the prescribed dose respectively was fulfilled in all cases.

The criterion of having at least $95 \%$ and $98 \%$ of CTV2 to receive at least $95 \%$ and $90 \%$ of the prescribed dose was not met in five and six patients respectively with an average of $92.8 \%$ of the volume received $95 \%$ of the dose and $97.3 \%$ of the volume received $90 \%$ of the dose. On the other hand the percentage of CTV3 who received $95 \%$ of dose had a wide range from $81 \%$ to $99 \%$ with an average of $88.3 \%$ and the percentage that received $90 \%$ of the dose ranged from $85 \%$ to $100 \%$ with an average of $94.5 \%$.

The maximum dose to the spinal cord was ranged from 50 to 39Gy (average 45.1Gy), however the dose to
$10 \%$ of the spinal cord volume ranged from 47 to $30 \mathrm{~Gy}$ (average 39.3Gy). On the other hand the dose to the brain stem was below 54Gy in all cases.

We succeed to keep the mean dose of both parotid glands below 26Gy in four patients and the other fifteen patients have at least one parotid gland below 26Gy.

During adjustments and fine tuning of the fields and segments we add 2 more small segments (total of 24 segments) in two patients planes to increase dose homogeneity. On the other hand we decreased the number of segments in one patient plane to a total of 19 segments this is because the spinal cord appears to be at one side of the CTV from some beam views.

Table 2a and $\mathbf{2 b}$ : The results of the FP-IMRT planning technique for 19 patients.

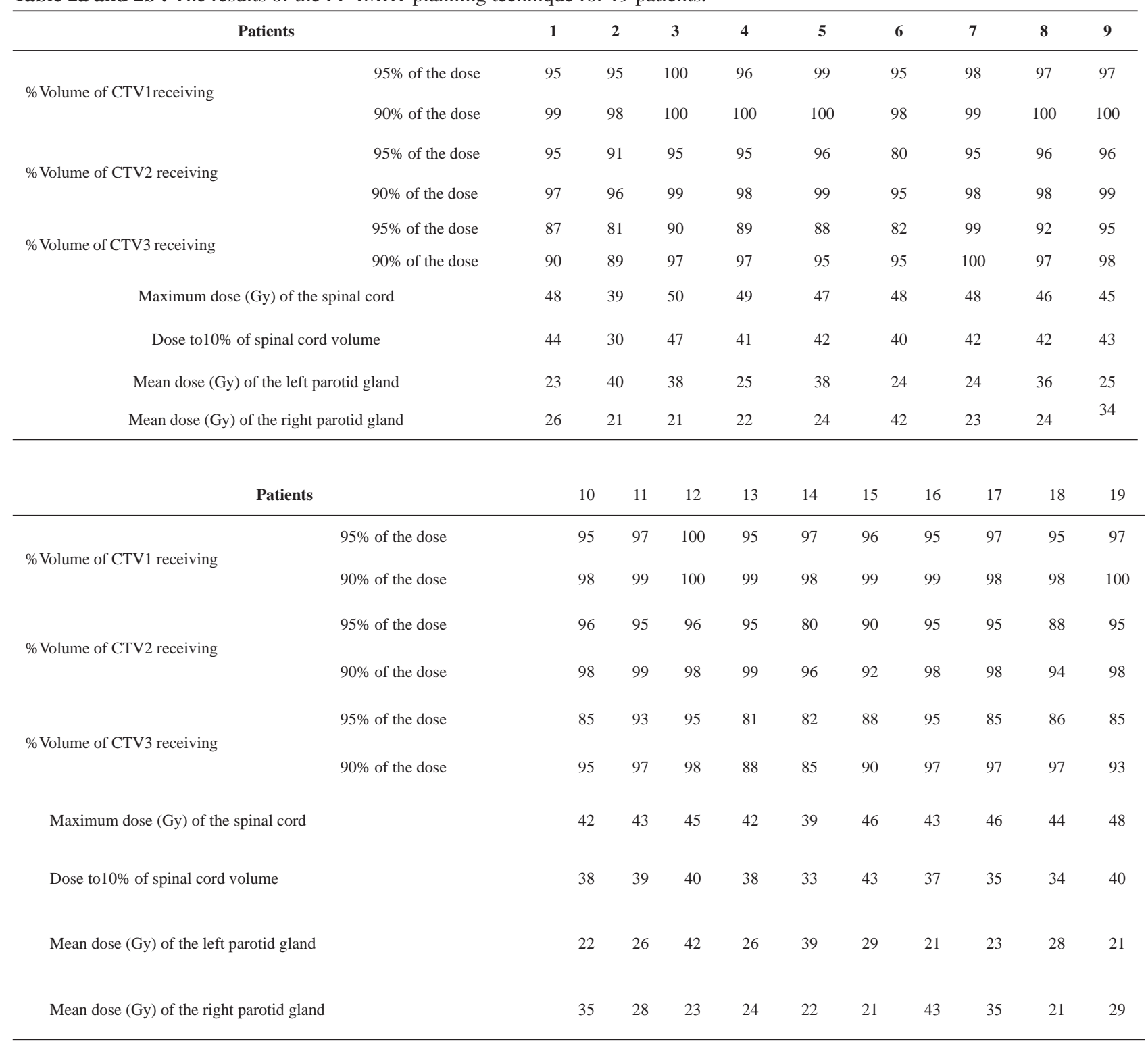


Figure (3) shows the isodose distributions on axial, sagittal and coronal images for a case of T2N1M0 nasopharyngeal carcinoma generated with the FPIMRT and (Fig. 4) shows the isodose distributions on axial, sagittal and coronal images for a case of T2N1M0 tonsillar carcinoma generated with the FP-IMRT. The red color indicates 66Gy, whereas the blue color indicates 60Gy and the yellow color indicates 54Gy. The isodose lines are displayed on an absolute dose scale.

The contours of the CTV1, CTV2 and the CTV3 are outlined in red, blue and rose colors, respectively. Notice that the CTV1 is completely encompassed by the 66Gy isodose line. The CTV2 (high-risk subclinical disease area) is encompassed by the 60Gy isodose line. CTV3 (elective nodal region) is encompassed by the 54Gy isodose line. No unexpected hot spots were found outside these target volumes. Notice the nice conformality of the isodose curves to the targets, whereas the right parotid gland in the first case and the left parotid gland in the second case were outside the 30Gy line and the majority of the left parotid in the first case was within the 30Gy line.

Figures. (5 and 6) show the dose-volume histogram of the two cases presented above (nasopharyngeal carcinoma and tonsillar carcinoma) treated with FP-IMRT technique. The CTV1, CTV2 and CTV3 had a good coverage, the dose to the spinal cord and brain stem; remain below the tolerance and one of the parotid glands has a mean dose below 26 gray in each case.

\section{Patient outcomes:}

All patients were assessed one month after completion of therapy; this assessment includes physical examination, CT scan or MRI of the primary and neck nodes. Endoscopy and biopsy was performed from any suspicious lesions.

Concerning the response to treatment, we found that 16 out of 19 achieved complete response (84.2\%).
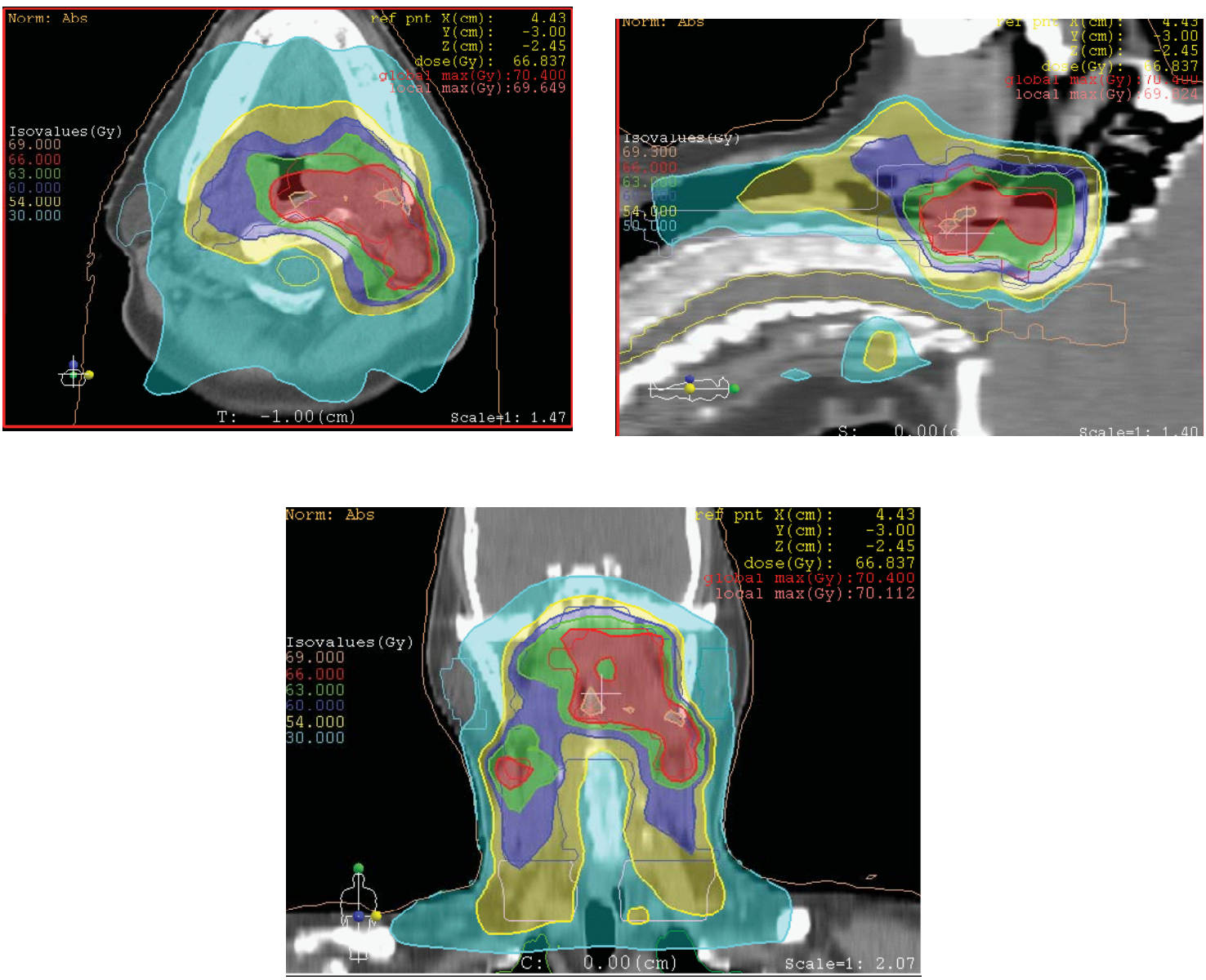

Fig. 3: Displays the axial, sagittal, and coronal slices of a patient with nasopharyngeal carcinoma, along with the isodose distributions. 


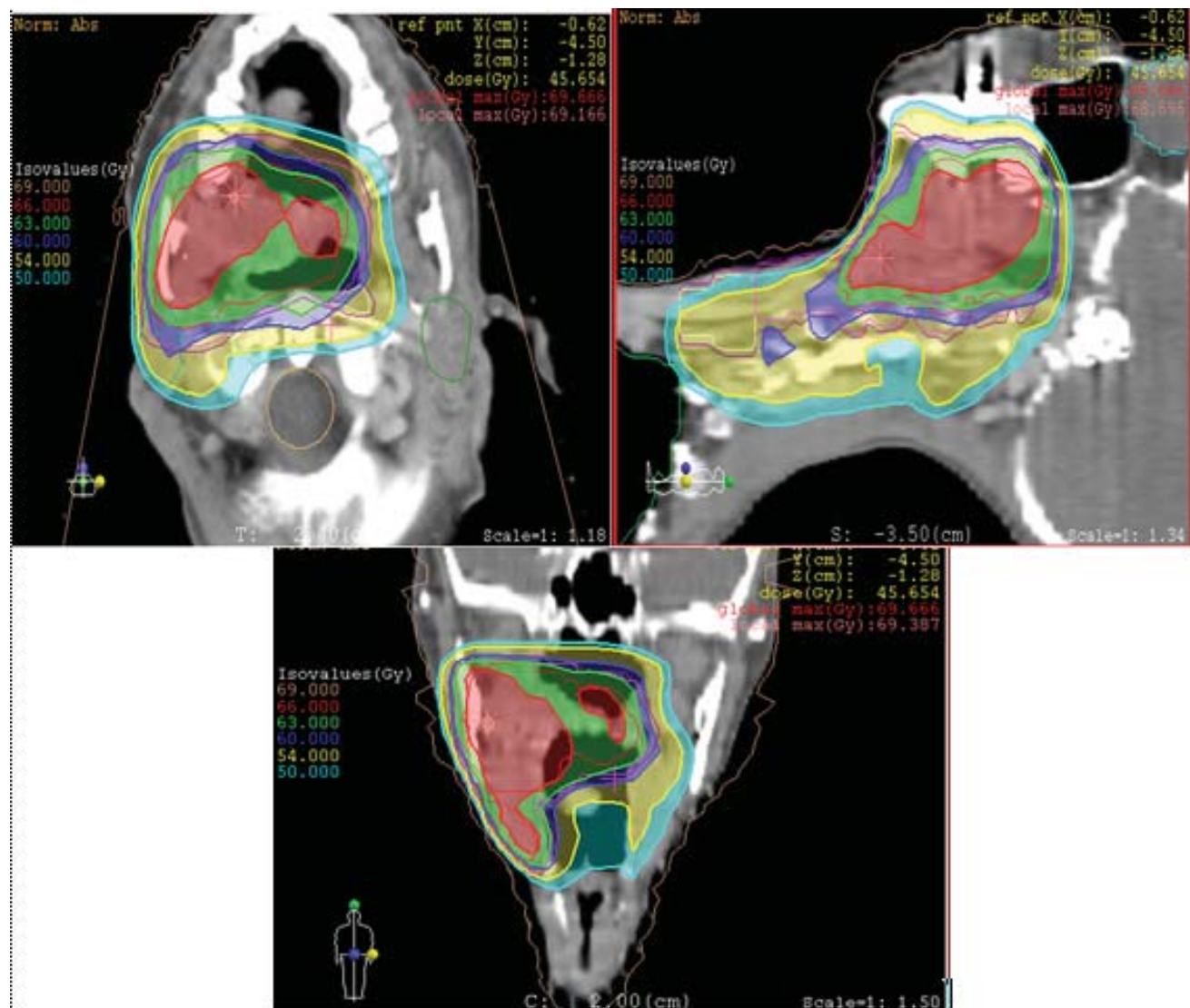

Fig. 4: Displays the axial, sagittal and coronal slices of a patient with tonsillar carcinoma, along with the isodose distributions.

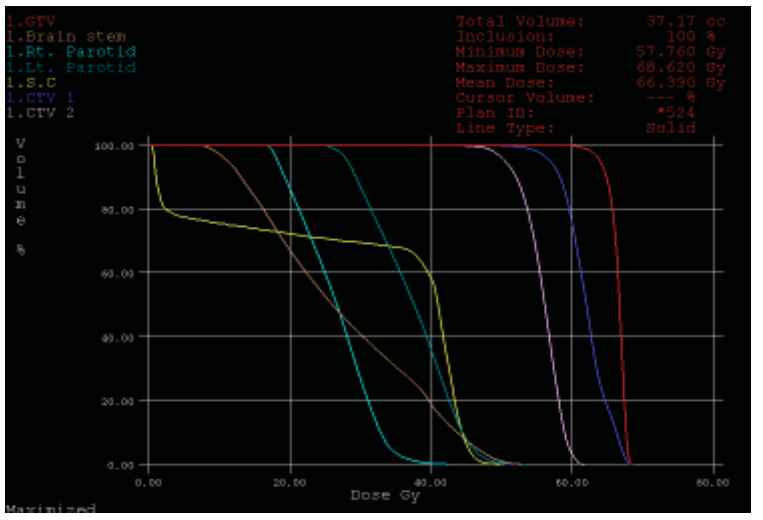

Fig. 5: Shows the dose-volume histogram of one patient with nasopharyngeal carcinoma it include the CTV1, CTV2, CTV3, spinal cord, brain stem, right and left parotid.

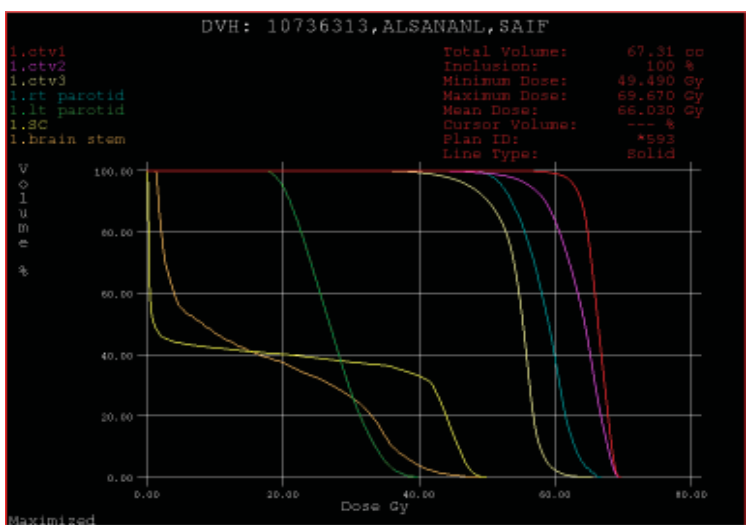

Fig. 6: Shows the dose-volume histogram of one patient with tonsillar carcinoma it include the CTV1, CTV2, CTV3, spinal cord, brain stem, right and left parotid.

\section{DISCUSSION}

Advancements in technology have made it possible to treat many complicated and irregularly shaped headand neck tumors ${ }^{16}$, further advances in 3D-CRT, such as IMRT, allow even better coverage of the tumor target while minimizing the dose delivered to the normal tissues. IMRT can be divided into two broad categories, forward planning vs. inverse planning. In forward planning, the planning dosimetrist or physicist selects the number, energy, weighting and angle of beams. The computer then calculates the dose distribution and generates beam's-eye views, along with dose-volume histograms. The plan is optimized by manual iteration or trial and error. This is in contrast to inverse planning, where one begins by defining the desired dose to the target and the normal tissues and the computer with its optimization program will go through multiple iterations seeking to find the best beam parameters that will yield the desired dose distribution ${ }^{17}$.

Recently, several articles have addressed the physical as well as clinical advantage of IP-IMRT over FP-IMRT or conventional radiotherapy. Since the introduction of IP-IMRT, difficult and advanced head-and-neck tumors that are impossible to cover adequately without exceeding the tolerance of the respective normal tissues are now possible to cover adequately with IP-IMRT. This is true for sites including nasopharynx, oropharynx and larynx. The conclusion has always been similar, that IP-IMRT 
achieved better coverage, though more inhomogeneous, when compared to conventional treatment techniques. In addition, there is always more sparing of surrounding normal tissues ${ }^{18}$. However, not every center has a dedicated team of physicists, dosimetrists and therapists that can handle large volumes of IP-IMRT patients. Without this dedicated team approach, IP-IMRT for complex head-and neck treatment planning may be nearly impossible. In addition, the prolonged treatment time for each IP-IMRT may not be feasible in many busy radiotherapy clinics. Therefore, given the above factors and because an excellent dose distribution covering the tumor target while sparing the nearby normal tissues can be achieved with the FP-IMRT technique ${ }^{12}$, it may be more efficient to treat patients with FP- IMRT until IPIMRT becomes feasible in a busy clinic.

The main point of discussion from the data reported here concerns the ability to reach dose objectives that are not in general achievable with conventional conformal planning techniques. The method described here offers the option of specifying different dose limits for the tumor (CTV1), areas at risk (CTV2-CTV3) and organ at risk. We succeed to achieve the desired dose target for CTV1, however we can not reach our aim in some cases as regard the CTV2 and CTV3, but even if we compare the average of the percentage of volume that receive $90 \%$ of the dose for CTV2 and CTV3 with our aim to reach $98 \%$ of the volume we found that the difference was $0.7 \%$ and $3.5 \%$, respectively, which to some extend acceptable especially that if we examine the dose volume histogram of those cases, we found that if we reduced the desired dose by $2 \%$ to $3 \%$ we found that $100 \%$ of the volume will be fully covered by this dose.

In our study, the major aim for the parotid glands was to achieve a mean dose below 26Gy as proposed by Eisbruch et al. ${ }^{19}$ We considered a mean dose of 26Gy as sufficient to substantially spare the gland function and we succeed to keep the mean dose of both parotid glands below 26Gy in four patients and the other fifteen patients have at least one parotid gland with a mean dose below 26Gy. However, the normal tissue complication probability (NTCP) for the parotid glands published by Eisbruch et $\mathrm{al}^{19}$. and another Group ${ }^{20}$ are continuous and monotonously increasing functions of the mean dose with a steep gradient between 20 and 30Gy. Any significant reduction of the mean dose in this range may thus be of clinical importance. Which means that even the cases that have a mean dose reaching up to 30Gy to one of the parotid gland, does not mean that this parotid gland is completely lost.

Because FP-IMRT and IP-IMRT have very sharp dose falloff gradients between the target and surrounding normal tissue, adequate target volume delineation is absolutely essential. Therefore, no matter what technology or excellent supporting staff that one center has, precise target volume delineation is absolutely critical. The treatment planning system will not treat areas not drawn on the CT slices, so the target volumes of each case are carefully and accurately defined jointly by the multidisciplinary team.

\section{CONCLUSION}

The forward-planning intensity-modulated radiation therapy (FP- IMRT) allows the delivery of a high dose to the target coverage while minimizing the normal tissue toxicity, In a center without the capabilities of IP-IMRT or in situations where patients are not ideal candidates for that technique, FP- IMRT can be useful and can achieve a good dose distribution.

\section{REFERENCES}

1. Gregoire V, De Neve W, Eisbruch A, Lee N, Van den Weyngaert D, Van Gestel D. Intensity-modulated radiation therapy for head and neck carcinoma. Oncologist 2007 May;12(5):555-64.

2. Mell LK, Roeske JC, Mundt AJ. A survey of intensity-modulated radiation therapy use in the United States. Cancer 2003 Jul 1;98(1):204-11.

3. De Gersem W, Claus F, De Wagter C, De Neve W. An anatomybased beam segmentation tool for intensity-modulated radiation therapy and its application to head-and-neck cancer. Int.J.Radiat. Oncol.Biol.Phys. 2001 Nov 1;51(3):849-59.

4. Brahme A. Optimized radiation therapy based on radiobiological objectives. Semin.Radiat.Oncol. 1999 Jan;9(1):35-47.

5. Lee N, Xia P, Fischbein NJ, Akazawa P, Akazawa C, Quivey JM. Intensity-modulated radiation therapy for head-and-neck cancer: The UCSF experience focusing on target volume delineation. Int.J.Radiat.Oncol.Biol.Phys. 2003 Sep 1;57(1):49-60.

6. Eisbruch A, Kim HM, Terrell JE, Marsh LH, Dawson LA, Ship JA. Xerostomia and its predictors following parotid-sparing irradiation of head-and-neck cancer. Int.J.Radiat.Oncol.Biol. Phys. 2001 Jul 1;50(3):695-704.

7. Xia P, Fu KK, Wong GW, Akazawa C, Verhey LJ. Comparison of treatment plans involving intensity-modulated radiotherapy for nasopharyngeal carcinoma. Int.J.Radiat.Oncol.Biol.Phys. 2000 Sep 1;48(2):329-37.

8. Cozzi L, Fogliata A, Bolsi A, Nicolini G, Bernier J. Threedimensional conformal vs. intensity-modulated radiotherapy in head -and-neck cancer patients: Comparative analysis of dosimetric and technical parameters. Int.J.Radiat.Oncol.Biol. Phys. 2004 Feb 1;58(2):617-24.

9. Pirzkall A, Carol M, Lohr F, Hoss A, Wannenmacher M, Debus J. Comparison of intensity-modulated radiotherapy with conventional conformal radiotherapy for complex-shaped tumors. Int.J.Radiat.Oncol.Biol.Phys. 2000 Dec 1;48(5):1371-80.

10. Chao KS, Ozyigit G, Blanco AI, Thorstad WL, Deasy JO, Haughey $\mathrm{BH}$, et al. Intensity-modulated radiation therapy for oropharyngeal carcinoma: Impact of tumor volume. Int.J.Radiat. Oncol.Biol.Phys. 2004 May 1;59(1):43-50.

11. Lee N, Akazawa C, Akazawa P, Quivey JM, Tang C, Verhey LJ, et al. A forward-planned treatment technique using multisegments in the treatment of head-and-neck cancer. Int.J.Radiat.Oncol.Biol. Phys. 2004 Jun 1;59(2):584-94. 
12. Sultanem K, Shu HK, Xia P, Akazawa C, Quivey JM, Verhey LJ, et al. Three-dimensional intensity-modulated radiotherapy in the treatment of nasopharyngeal carcinoma: The University of California-San Francisco experience. Int.J.Radiat.Oncol.Biol. Phys. 2000 Oct 1;48(3):711-22.

13. Mohan R, Wu Q, Manning M, Schmidt Ullrich R. Radiobiological considerations in the design of fractionation strategies for intensity-modulated radiation therapy of head and neck cancers. Int.J.Radiat.Oncol.Biol.Phys. 2000 Feb 1;46(3):619-30.

14. Clifford Chao KS, Apisarnthanarax S, Ozyigit G. Practical essentials of intensity modulated radiation therapy. 2nd ed.: Lippincott Williams \& Wilkins; 2004.

15. Apisarnthanarax S, Elliott DD, El Naggar AK, Asper JA, Blanco A, Ang KK, et al. Determining optimal clinical target volume margins in head-and-neck cancer based on microscopic extracapsular extension of metastatic neck nodes. Int.J.Radiat. Oncol.Biol.Phys. 2006 Mar 1;64(3):678-83.

16. Lee N, Xia P, Quivey JM, Sultanem K, Poon I, Akazawa C, et al. Intensity-modulated radiotherapy in the treatment of nasopharyngeal carcinoma: An update of the UCSF experience. Int.J.Radiat.Oncol.Biol.Phys. 2002 May 1;53(1):12-22.

17. Nutting C, Dearnaley DP, Webb S. Intensity modulated radiation therapy: A clinical review. Br.J.Radiol. 2000 May;73(869):459-69.

18. Hunt MA, Zelefsky MJ, Wolden S, Chui CS, LoSasso T, Rosenzweig K, et al. Treatment planning and delivery of intensitymodulated radiation therapy for primary nasopharynx cancer. Int.J.Radiat.Oncol.Biol.Phys. 2001 Mar 1;49(3):623-32.

19. Eisbruch A, Ten Haken RK, Kim HM, Marsh LH, Ship JA. Dose, volume and function relationships in parotid salivary glands following conformal and intensity-modulated irradiation of head and neck cancer. Int.J.Radiat.Oncol.Biol.Phys. 1999 Oct 1;45(3):577-87.

20. Roesink JM, Moerland MA, Battermann JJ, Hordijk GJ, Terhaard $\mathrm{CH}$. Quantitative dose-volume response analysis of changes in parotid gland function after radiotherapy in the head-and-neck region. Int.J.Radiat.Oncol.Biol.Phys. 2001 Nov 15;51(4):938-46. 